\title{
One-dimensional DCNN Feature Selective Transformation with LSTM-RDN for Image Classification
}

\author{
Chaorong Li, Yuanyuan Huang, Wei Huang, Fengqing Qin
}

\begin{abstract}
Feature selection and transformation are the important techniques in machine learning field. A good feature selection or transformation will greatly improve the performance of classification method. In this work, we proposed a simple but efficient image classification method which is based on twostage processing strategy. In the first stage, the one-dimensional features are obtained from image by transfer learning with the pre-trained Deep Convolutional Neural Networks (DCNN). These one-dimensional DCNN features still have the shortcomings of information redundancy and weak distinguishing ability. Therefore, it is necessary to use feature transformation to further obtain more discriminative features. We propose a feature learning and selective transformation network based on Long Short-Term Memory (LSTM) combing ReLU and Dropout layers (called LSTM-RDN) to further process onedimensional DCNN features. The verification experiments were conducted on three public object image datasets (Cifar10, Cifar100 and Fashion-MNIST), three fine-grained image datasets (CUB200-2011, Stanford-Cars, FGVC-Aircraft) and a COVID19 dataset, and several backbone network models were used, including AlexNet, VGG16, ResNet18, ResNet101, InceptionV2 and EfficientNet-b0. Experimental results have shown that the recognition performance of the proposed method can significantly exceed the performance of existing state-of-the-art methods. The level of machine vision classification has reached the bottleneck, it is difficult to solve this problem by using a large-scale network model which has huge parameters that need to be optimized. We present an effective approach for breaking through the bottleneck of visual classification task by feature extraction with backbone DCNN and feature selective transformation with LSTM-RDN, separately. The code and pre-trained models are available from: https://github.com/lilllllllI/LSTM-RDN
\end{abstract}

Index Terms-Feature Selective Transformation, LSTM, Image Classification,COVID-19, Deep Convolutional Neural Network.

This work is supported by Project of Sichuan Science and Technology Program (No.2019YFS0068), Projects of Sichuan Provincial Department of Education (Nos.18ZA0538,17ZA0452), Project of Yibin science and Technology Bureau(No.2017ZSF009-9), Projects of Yibin University (Nos.2020YY02,2021YY06). (Corresponding author: Chaorong Li)

C.R. Li is with the School of Computer, Yibin University, he is work for Chongqing University of Technology (e-mail: lichaorong88@163.com).

Y.Y. Huang is with the Department of cyberspace engineering, chengdu university of information technology, Chengdu 610225, China (e-mail: iyyhuang@hotmail.com).

W. Huang is with The Clinical Hospital of Chengdu Brain Science Institute, MOE Key Lab for Neuroinformation, University of Electronic Science and Technology of China, Chengdu 610054, China (e-mail: lembert1990@163.com).

F. Qin is with the Department of Computer Science and Information Engineering, Yibin University, Yibin 644000, China (e-mail: qinfengqing@163.com).

Manuscript received xxxx xx, 2020; revised xxxx xx, 2020.

\section{INTRODUCTION}

Machine vision technique is becoming more and more important in our life and work which in turn promotes the development of machine vision technique. In fact, our requirements for the technical level of machine vision are getting higher and higher. For example, we require computers to accurately identify animal types, accurately locate moving targets, and accurately predict the type of pneumonia through CT images. There are three major tasks of machine vision including image classification, target detection, and image segmentation. Image classification is the basis among the three tasks. Its importance is self-evident, but the current image classification technology is still at a low level and cannot meet the requirements of our life and work.

In machine vision, the feature extraction of the image is a necessary step. Filters and descriptors[1] are two basic techniques for extracting image features. The former mainly uses linear transformation of pixels in the local area of the image to obtain more prominent image information and meanwhile remove the noise of image. These filters include DoG[2], Wavelet[3], etc. Descriptors such as LBP[4] and SIFT[5], mainly perform more complex calculations on the pixels within the local area of the image, and these calculations include calculating the local image geometric information or statistical information. Due to the complexity of image, the local features yielded from the aforementioned handcrafted descriptors or filters are mainly determined by the pixels in the local area, and their performance is very limited. In recent years, researchers' interest has shifted from handcrafted descriptors and filters to learning-based descriptors and filters on machine learning filed. The latter introduces a learning mechanism to integrate the local features obtained from local regions into global features, and at the same time adjusts the parameters of filters or descriptors through training feedback information to obtain better representation performance. Dictionary learning [6],[7], Bag of Visual Words(BoVW) [8] and neural network learning[9] are three types of learning-based descriptors.

Filters and descriptors focus on the local features of the image, and then use statistical techniques such as histograms to get the overall features, while the method based on Deep Convolutional Neural Network (DCNN) directly extracts the overall features from the whole image. The advantage of this direct feature extraction method is that it can implement an end-to-end learning process, and it is more convenient to 
obtain the features of high-level image semantics. AlexNet[10] is a classic shallow and single-branch $\mathrm{CNN}$; it outperformed the traditional machine learning methods and won the championship on the ImageNet dataset in 2012. With the development of the times, this shallow and single-branch neural network is increasingly unable to meet the challenge of more complex applications. Since then, various effective network models have been designed to overcome the difficulties encountered in image classification. At present, the neural network structure is developing in the direction of more network branches (horizontal) and deeper network layers (vertical). The vertical development of the network focuses on using deeper layers to obtain better results. The original AlexNet only had more than 20 layers, later networks such as VGG model[11] have dozens of layers, and current networks such as NasNet[12] reaches to thousands of layers. The horizontal development of the network is not as fast as the vertical direction; increasing limited branches at certain layer of the network to extract a wider range of image features is the main strategy; the models have more branches including the residual structure of the ResNet[13], WideResNet[14] and the branch structure of the Inception networks[15], [16], [17].

The DCNN image classification performance has reached a bottleneck, and it is very difficult to significantly improve the recognition performance. Recently, some new visual models appeared such as Transformer and multi-layer perception (MLP) model. Transformer[18] used in the natural language processing field, has been introduced into the field of machine vision and received great attention. ResMLP[19] and MLPMixer[20] are two simple MLP-based network and achieve unexpectedly high performance on ImageNet classification benchmarks; they have an architecture built entirely upon MLP which are embedded into the residual network.

Super-deep (DCNN) and multi-branched network structure make the model more demanding on the machine hardware. It usually takes several days or even dozens of weeks to train a large model because the huge parameters of network; but the performance improvement is indeed insignificant, at least not achieving our expectation. Is this kind of super-deep structure necessary? Researchers are exploring using Transformers, Attention-based models[21], MLP-based networks, or different combinations of these methods to avoid the disadvantages of super-deep network model. Different from these methods, we use two-stage processing strategy to overcome the problem of huge parameters, so as to improve the performance of network model. The proposed method only needs the one-dimensional features of the DCNN, such as ResNet18, and then uses the feature selective transformation techniques and fine-tunes the model parameters, to obtain promising results which are much higher than the recognition results of other super-deep DCNN and Transformer models.

Our experiments show that there is no significant performance improvement between the shallow neural network and the super-deep neural network after feature selective transformation at most of cases, which is also one of the main contributions of this article. In addition, because there is correlation between DCNN features, we regard the one-dimensional DCNN output features as vector sequences, and use Short-
Term Memory (LSTM) to perform feature transformation to make the features have better distinguishing capabilities; at the same time, we also added ReLU and Dropout functions in the network to perform feature selective transformation for improving the generalization ability.

\section{RELATED WORK}

A typical classification task of machine vision is to predict the labels through sample images. The first step of classification task is to perform the feature extraction which is to convert the sample image into a set of features with obvious physical meaning such as geometric and texture features, or statistical significance(see Fig 1). If the samples have few features, we will consider adding more features. In reality, there are often too many features, and some of them need to be reduced. Therefore, we often use feature selection or feature transformation to continue the original features. Feature transformation and selection are to find the most effective features (in-variance of similar samples, discrimination of different samples, robustness to noise) from the original features. They not only alleviate overfitting, the runtime and improve the generalization ability of the model, but also enable the model to obtain better interpret-ability and enhance the understanding content of the images. Typical feature extraction algorithms

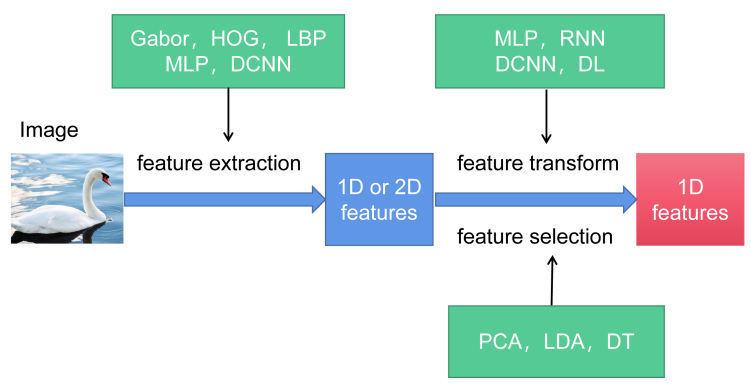

Fig. 1. Feature extraction, feature transformation and feature selection.

include Gabor [22], HOG[23] and LBP descriptors used to obtained the low-level features of image; MLP[24] and DCNN which is used to extract the high-level features. Feature transformation methods are relatively extensive, and involve most of the machine learning and pattern recognition approaches. Some feature extraction methods can also be used as feature transformation like MLP and DCNN. In addition, Recurrent Neural Network (RNN)[25] and Dictionary Learning (DL)[26] is also commonly used for feature transformation. Feature selection refers to selecting $N$ significant features from the existing $M$ features to optimize the specific indicators of the system. Principal component analysis (PCA)[27] and linear discriminant analysis (LDA)[28] and Deep Forest (DF) 29] are the effective methods for feature selection. It should be emphasized that the difference between the three technologies (feature extraction, transformation and selection) is not clear. In many cases, the same model (such as DCNN) can be used for feature extraction and feature transformation; PCA and LDA also use feature selection operation when performing feature transformation. For the sake of distinction in this work, the method of finding the optimal features by using feature 
transformation and selection at the same time is called the feature selective transformation.

In recent years, LSTM network have emerged for image classification and other processing. It is a time cyclic neural network, which is specially designed to solve the longterm dependency problem of general RNN (Recurrent Neural Network). RNN has a chain form of repeated neural network modules. In a standard RNN, this repeated structural module is a very simple module, such as a Tanh layer or Linear Layer; while the repeating module of LSTM is more complex and much more effective. The basic cell of LSTM is shown in Fig. 2. Since LSTM is a kind of RNN, its output is affected by the current input $x_{t}$, the previous input $h_{t-1}$ (hidden units), and the influence of the cell state $C_{t-1}$. Overall, LSTM consists of three gates (forget gate, input gates and output gate) which combine the inputs and control the output. The forget gate is expressed as follows

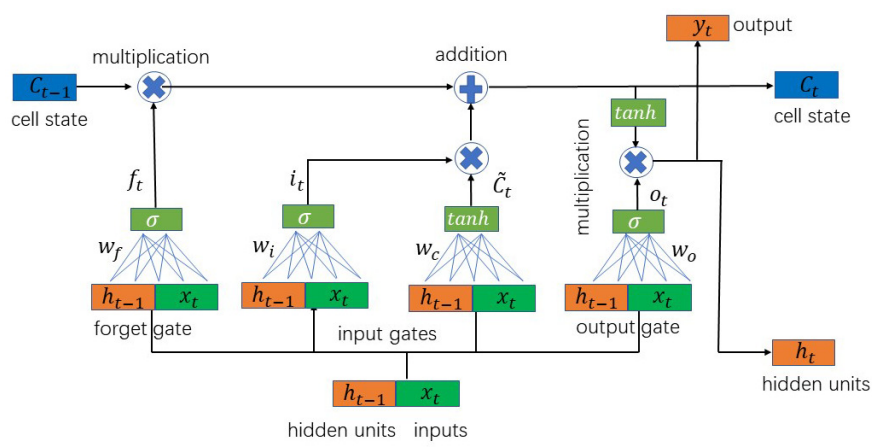

Fig. 2. Internal structure of LSTM cell.

$$
f_{t}=\sigma\left(W_{f} \cdot\left[h_{t-1}, x_{t}\right]+b_{f}\right)
$$

There are two important parts in the input gates which will update the cell state, denoted as follows.

$$
\begin{aligned}
i_{t} & =\sigma\left(W_{i} \cdot\left[h_{t-1}, x_{t}\right]+b_{f}\right), \\
\tilde{C} & =\tanh \left(W_{C} \cdot\left[h_{t-1}, x_{t}\right]+b_{C}\right)
\end{aligned}
$$

The new cell state $C_{t}$ is expressed as

$$
C_{t}=f_{t} * C_{t-1}+i_{t} * \tilde{C}_{t}
$$

The final output $h_{t}$ (or $y_{t}$ ) is the result of multiplying the output $o_{t}$ of the output gate and the current cell state $C_{t}$

$$
\begin{aligned}
o_{t} & =\sigma\left(W_{o}\left[h_{t-1}, x_{t}\right]+b_{o}\right), \\
h_{t} & =o_{t} * \tanh \left(C_{t}\right)
\end{aligned}
$$

In image analysis, although DCNN has achieved great success, its performance is still difficult to meet the requirements in practical applications. LSTM is an effective tool to make up for the shortcomings of DCNN. Recently, there have been many examples of DCNN combined with LSTM. For example, [30] proposes handwriting recognition based on Convolutional-LSTM Network; [31] proposes Tree-Structured Regional CNN-LSTM model for dimensional sentiment analysis. In the hybrid combination, LSTM is mostly placed at the end of the network or used as the intermediate processing unit of the network.

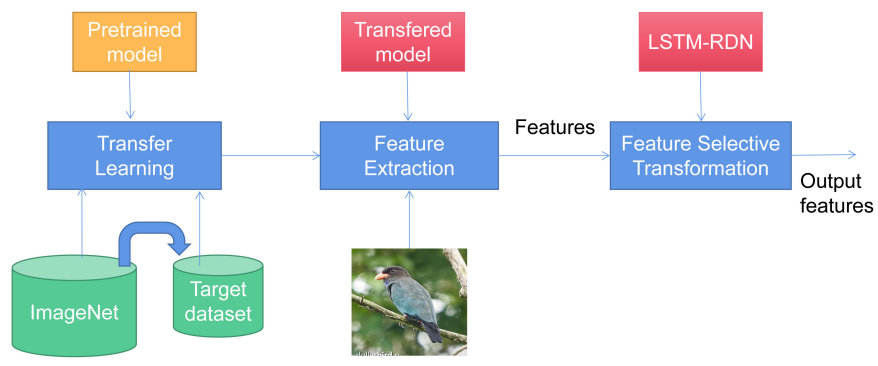

Fig. 3. The flowchart of the selective feature transformation for image classification.

\section{LSTM-BASED FeAture SElective TRANSFORMATION NETWORK}

The process of the proposed image recognition method based on two-stage strategy with feature selective transformation is shown in Fig 3. The method uses a pre-trained network model (such as ResNet) as the backbone model, and then train the pre-trained network model on a specific image dataset by using transfer learning for obtaining the specified network model. By using the specified network model, we can obtain the two new one-dimensional DCNN datasets: train set and test set. Finally, we use the feature selection network based on three types of components LSTM, ReLU and Dropout layers (called LSTM-RDN) to train on the new train set and classify the test images on the new test set.

LSTM-RDN roughly consists of two parts, as shown in Fig 4. The first part is the feature transformation component which is a LSTM layer. We regard the one-dimensional DCNN features as a sequence, and there is a strong correlation between the features. This correlation can be memorized and utilized by LSTM to optimize the transformation weights of the current input features. The second part is a feature selection component composed of a Rectified Linear Unit(ReLU) layer and several Dropout layers. This part uses ReLU to perform nonlinear mapping on the output of the LSTM layer, and then uses three Dropout layers to discard some features.

The two parts of the proposed network are mainly to improve the discriminability of DCNN features as much as possible. The following experiments show that if only LSTM layer is used without the feature selection part (ReLU and Dropout layers), the model's performance will be greatly reduced (See the experiment and discussion part for details). At the front of LSTM-RDN is the sequence layer, which is used to organize the serialized data, followed by a Dropout layer (named D0) which is used to randomly discard some features before the DCNN features are fed to the LSTM layer.

The only parameter of the Dropout layer is the drop probability, which plays a very important role. Probability for dropping out input elements, specified as a numeric scalar in the range [0,1]. In LSTM-RDN, we set up a total of three Dropout layers before and after the LSTM layer. Theoretically, D1 and D2 can be combined into one Dropout. Suppose the dropping probabilities of D1 and D2 are $p_{1}$ and $p_{2}$, 


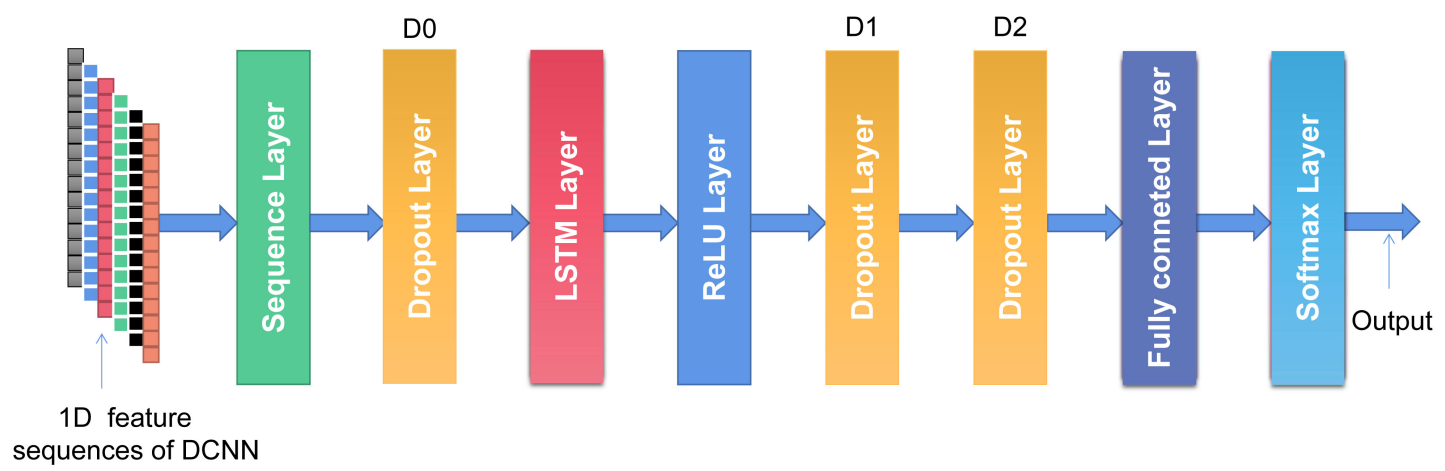

Fig. 4. LSTM-RDN for selective feature transformation. LSTM-RDN contains a LSTM, the ReLU and Dropout layers. The part of selective feature transform consists of the ReLU layer and the three Dropout layers (D0,D1 and D2) .

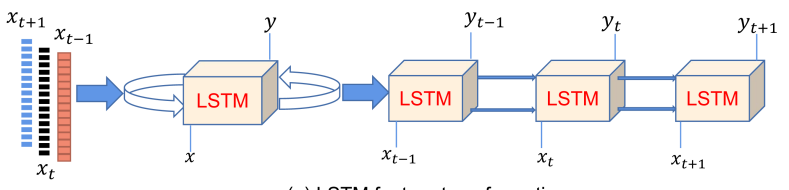

(a) LSTM feature transformation

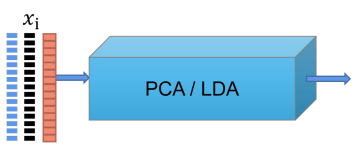

(b) PCA / LDA feature transform

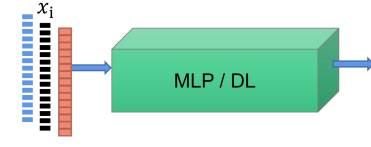

(c) MLP and DL feature transform
Fig. 5. The differences between LSTM feature transform and other methods.

respectively, and their combined dropping probability $p$ is

$$
p=1-\left(1-p_{1}\right) \times\left(1-p_{2}\right),
$$

Because the drop probability is a very critical parameter in LSTM-RDN, its subtle changes may cause large fluctuations in the recognition performance of the model. Therefore, we split a dropout layer into several layers for the purpose of fine-tuning the parameters. Similarly, we can divide dropout D0 into multiple dropouts using EQ 5 .

LSTM feature transformation has the similar function as PCA, LDA, Dictionary Learning and fully connected networks, all of which can transform DCNN feature vectors into new feature vectors with more distinguishing performance. PCA / LDA needs to compute the divergence matrix of the all the DCNN feature vectors in the training set at one time; however, the performance is limited because of the lack of learning mechanisms. Fully connected network / dictionary learning obtains the transform model / dictionary by sending the samples to the model one by one or batch by batch with learning approach, but does not consider the correlation between the feature sequences. The characteristic of LSTM feature transformation is that LSTM processes onedimensional DCNN features as time series, so it inputs these features one by one to train the model (see Fig 5 ), and at the same time it computes the output with the current and previous states of the model.

The schematic diagram of the feature selection part composed of a ReLU and three Dropout layers is given in Fig 6 .
ReLU is a commonly used activation function in artificial neural networks. The reason why ReLU is very useful is that it makes the output of some neurons zero, which leads to the sparsity of feature sparsity (or network). That is, it can let each neuron perform the screening function: if the input of neuron is larger than the given value, then the input will be enlarged vigorously; if not it will be cut off, denoted by

$$
f(x)=\max \left(0, z_{i}\right)
$$

where $z_{i}$ is the input of neuron in the ReLU layer. ReLU and Dropout implement similar functions in different ways. Dropout is to randomly drop some nodes (or weight parameters) to participate in network training, so as to obtain more robust new features. Since both ReLU and Dropout have the function of zeroing or discarding a certain feature, we call their function here as feature selective transformation. "Attention" mechanism which is a popular technique uses weight to amplify or weaken the value of certain input variables or the block of feature map [32]. We proposed LSTM-RDN also has similar select functions as Attention mechanism.

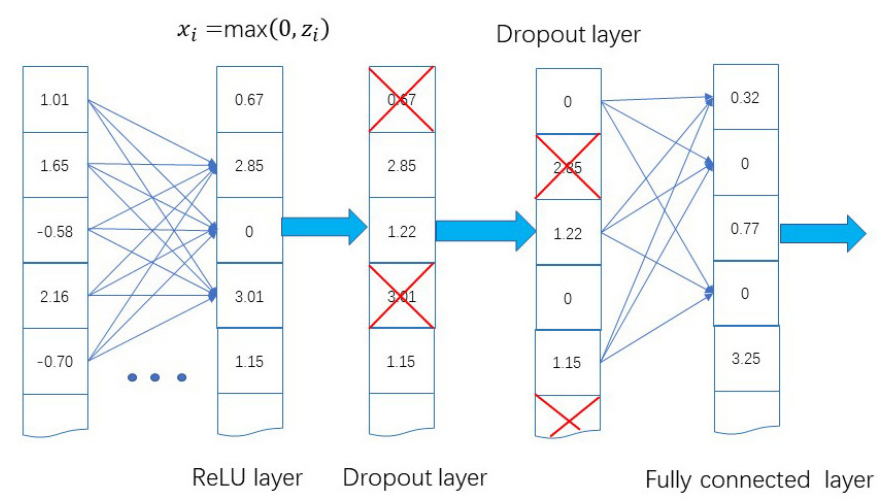

Fig. 6. The detail of feature selection based on ReLU and Dropout layers.

\section{EXPERIMENTS}

The experiment was conducted on two types of image datasets and a COVID-19 dataset. The first type is object datasets, including Cifar10, Cifar100 and fashion-MNIST. The second type is fine-Grained image datasets, including 


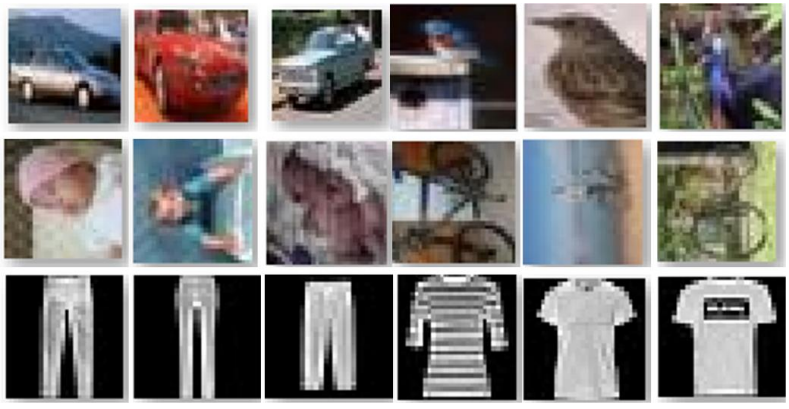

Fig. 7. Samples selected from Cifar10(the first row), Cifar100(the second row) and Fashion MNIST(the third row)

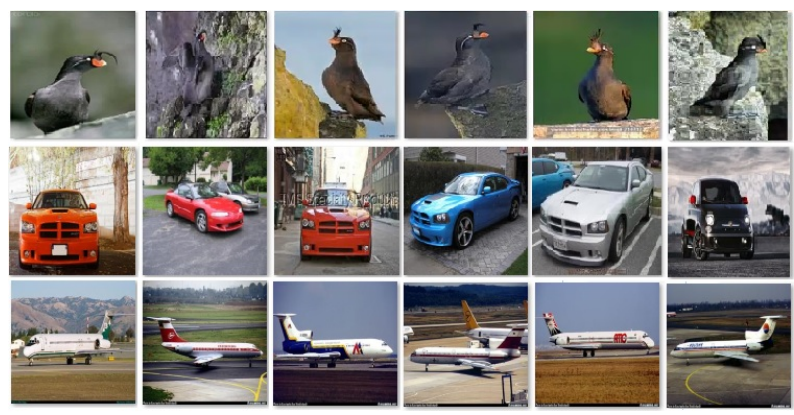

Fig. 8. Samples selected from CUB200-2011(the first row), Stanford-Cars(the second row) and FGVC-Aircraft(the third row)

CUB200-2011, Stanford Cars and FGVC-Aircraft. Some samples selected from these datasets are shown in Fig 7 and Fig 8

The backbone network models we used include: AlexNet, VGG16, ResNet18, ResNet101, InceptionV2 and EfficientNetb0. These pre-trained models are all trained on ImageNet. After downloading these models, we train them with transfer learning on the above datasets, respectively, to obtain the 1D DCNN features of the images. To object and fine-grained image recognition, in the feature selective transformation phase, Root Mean Square Prop (RMSProp) optimization algorithm is used to train LSTM-RDN; the initial learning rate is 0.001 , and the maximum number of initial iterations is set to 2000 (if the mini-batch loss is not less than 0.01 or the mini-batch recognition accuracy is less than $100 \%$, we will increase the number of training appropriately). Under normal circumstances, the dropout probability of several dropout layers is set to 0.5 ; when the number of training samples is small or the feature dimension is large (such as the 4096-dimensional features of VGG16), the dropout probability will be increased to 0.6-0.85.

For different databases and different backbone networks, we use fine-tuning approach to train LSTM-RDN model in the experiments. Due to the randomness in the training process of LSTM-RDN, the results of several tests may be different, and the classification accuracies of a model obtained by the experiment are the relatively stable results in several tests. Our experiments were performed on a computer with CPU i9 and GPU 2080ti environment.

\section{A. Object Classification}

The CIFAR-10 dataset consists of $6000032 \times 32$ color images in 10 classes, with 6000 images per class. There are 50000 training images and 10000 test images. Cifar100 has 100 classes containing 600 images each. There are 500 training images and 100 testing images per class. Fashion-MNIST dataset contains 10 categories of images. The training dataset contains 6000 samples for each category, the test dataset contains 1000 samples for each category, and there are 10 categories in total.

TABLE I

RECOGNITION RESULTS ON OBJECT DATASETS

\begin{tabular}{c|c|c|c|c}
\hline Dataset & DCNN & Transfer & LSTM-RDN & Imp. \\
\hline \multirow{5}{*}{ Cifar10 } & AlexNet & 85.81 & 99.93 & 14.12 \\
& VGG16 & 88.06 & $\mathbf{9 9 . 9 4}$ & 11.88 \\
& ResNet18 & 90.44 & 99.91 & 9.47 \\
& ResNet101 & 93.36 & 99.82 & 6.46 \\
& InceptionV2 & 93.58 & 99.93 & 6.19 \\
& EfficientNet-b0 & 89.95 & 99.39 & 9.44 \\
\hline \hline \multirow{5}{*}{ Cifar100 } & AlexNet & 62.33 & 96.57 & 34.24 \\
& VGG16 & 63.27 & $\mathbf{9 8 . 2 1}$ & 34.94 \\
& ResNet18 & 64.01 & 95.65 & 31.64 \\
& ResNet101 & 66.97 & 97.33 & 30.36 \\
& InceptionV2 & 68.28 & 98.17 & 29.89 \\
& EfficientNet-b0 & 64.6 & 95.29 & 30.69 \\
\hline \hline & AlexNet & 94.41 & $\mathbf{9 9 . 9 8}$ & 5.57 \\
& VGG16 & 94.91 & 99.95 & 5.04 \\
Fashion & ResNet18 & 94.37 & 99.84 & 5.47 \\
& ResNet101 & 94.98 & 99.89 & 4.91 \\
& InceptionV2 & 94.09 & 99.75 & 5.66 \\
& EfficientNet-b0 & 93.12 & 99.75 & 6.63 \\
\hline
\end{tabular}

After the pre-trained model is performed with transfer learning on target dataset, we compute the recognition accuracies of backbones by using the transfer-learned models which classify the images with Softmax layer, and the recognition accuracies of our proposed LSTM-RDN based on the 1D features of the transfer-learned models. The recognition accuracies are listed in Table I. On the three datasets of Cifar10, Cifar100 and Fashion-MNIST, the recognition accuracies of our method on three datasets are higher than $98 \%$, and the highest is 99.98\% on Fashion-MNIST. The performance of these pretrained models has been significantly improved after using LSTM-RDN, and the recognition accuracies on Cifar10 and Fashion-MNIST has been improved from 5\% to $14 \%$ (see the rightmost column of Table 2). On Fashion-MNIST, the recognition accuracies of these models exceed 99\%. Especially on Cifar100, the recognition accuracies of the transferlearned models are only 60\%, and LSTM-RDN can increase backbones' recognition accuracies to more than $95 \%$, and the improved percentage point is more than $30 \%$. To illustrate our method LSTM-RDN is effective and advanced, we compare it with the advanced methods that have been published. These comparisons are listed in Table $\mathrm{IT}$ TableIV Currently, the best records on the three datasets are: $99.70 \%$ (Cifar10), $96.08 \%(\mathrm{Ci}-$ far 100 ), 96.91\%(Fashion-MNIST), and our highest results are 99.94\% (Cifar10), 98.21\%(Cifar100) and 99.98\% (FashionMNIST). It is obvious that the recognition performance of LSTM-RDN based on pre-trained backbones is significantly higher than the best record. 
TABLE II

RECENT RESULTS ON CIFAR 10

\begin{tabular}{c|c|c}
\hline DCNN & Backbone model & Accuracy \\
\hline Our LSTM-RDN & AlexNet & 99.93 \\
Our LSTM-RDN & VGG16 & $\mathbf{9 9 . 9 4}$ \\
Our LSTM-RDN & ResNet18 & 99.91 \\
Our LSTM-RDN & ResNet101 & 99.82 \\
Our LSTM-RDN & InceptionV2 & 99.93 \\
Our LSTM-RDN & EfficientNet-b0 & 99.39 \\
\hline EffNet-L2[33] & ResNet & $\mathbf{9 9 . 7 0}$ \\
ViT-H[2]] & ResNet152 & 99.50 \\
EfficientNet-B7[34] & - & 98.90 \\
BiT-L[35] & ResNet & 99.30 \\
LaNet[36] & ResNet & 99.03 \\
MUXNets[37] & - & 98.0 \\
DAT[38] & AmoebaNet-B & 98.80 \\
NasMobile[39] & - & 91.88 \\
ResMLP-24[19] & MPL & 98.70 \\
\hline
\end{tabular}

TABLE III

RECENT RESULTS ON CIFAR 100

\begin{tabular}{c|c|c}
\hline DCNN & Backbone model & Accuracy \\
\hline Our LSTM-RDN & AlexNet & 96.57 \\
Our LSTM-RDN & VGG16 & $\mathbf{9 8 . 2 1}$ \\
Our LSTM-RDN & ResNet18 & 95.65 \\
Our LSTM-RDN & ResNet101 & 97.33 \\
Our LSTM-RDN & InceptionV2 & 98.17 \\
Our LSTM-RDN & EfficientNet-b0 & 95.29 \\
\hline EffNet-L2[33] & ResNet & $\mathbf{9 6 . 0 8}$ \\
TResNet[40] & ResNet & 91.15 \\
ViT-H[21] & ResNet152 & 94.55 \\
EfficientNet[34] & - & 91.70 \\
BiT-L[35] & ResNet & 93.51 \\
MUXNets[37] & - & 86.11 \\
DARTS[41] & ResNet110 & 84.10 \\
ResMLP-24[19] & MPL & 89.5 \\
\hline
\end{tabular}

\section{B. Fine-Grained Image Classification}

Fine-grained image recognition is a more refined distinction between target image categories, such as not only identifying the image label is a dog, but also telling whether it is a Labrador or a Husky. The task of fine-grained image recognition has been proposed in the past few years, This task has not been well achieved, on the contrary, it is far from our expectations.

CUB200-2011 dataset has 11788 bird images, including 200 bird sub-categories. The training dataset has 5994 images, and the test set has 5794 images. Stanford Cars dataset is an image dataset containing 196 types of cars. It is mainly

TABLE IV

RECENT RESULTS ON FASHION-MNIST

\begin{tabular}{c|c|c}
\hline DCNN & Backbone model & Accuracy \\
\hline Our LSTM-RDN & AlexNet & $\mathbf{9 9 . 9 8}$ \\
Our LSTM-RDN & VGG16 & 99.95 \\
Our LSTM-RDN & ResNet18 & 99.84 \\
Our LSTM-RDN & ResNet101 & 99.89 \\
Our LSTM-RDN & InceptionV2 & 99.75 \\
Our LSTM-RDN & EfficientNet-b0 & 99.75 \\
\hline \hline Few-shot Learning[42] & ResNet & 91.42 \\
DARTS[41] & ResNet110 & $\mathbf{9 6 . 9 1}$ \\
E2E-3M[43] & - & 95.92 \\
SpinalNet[44] & VGG-5 & 94.68 \\
Shake-Shake[33] & - & 96.41 \\
\hline
\end{tabular}

used for image classification. It has a total of 16185 images, of which 8144 and 8041 images are training images and test images. FGVC-Aircraft is a classic benchmark in fine-grained image classification. The dataset contains 10200 images of aircraft, with 100 images for each of 102 different aircraft model variants. The dataset is divided into training, validation and test subsets.

On Fine-grained datasets, the recognition accuracies of LSTM-RDN with different DCNN models are listed in Table $\mathrm{V}$. It can be seen that the classification ability of the pretrained model on these three fine-grained images is relatively poor, and the recognition accuracies of these models are roughly between $50 \%$ and $70 \%$. However, after feature selective transformation with LSTM-RDN, their recognition accuracies soared to more than $90 \%$, and the performance increased by 30 to $50 \%$. The results shows that LSTM-RDN is very effective.

TABLE V

RECOGNITION RESULTS ON FINE-GRAINED DATASETS

\begin{tabular}{c|c|c|c|c}
\hline Dataset & Model & Transfer & LSTM-RDN & Imp. \\
\hline & AlexNet & 47.98 & 92.30 & 44.32 \\
& VGG16 & 59.21 & 96.27 & 37.06 \\
CUB200 & ResNet18 & 62.72 & 98.11 & 35.39 \\
-2011 & ResNet101 & 68.63 & $\mathbf{9 8 . 1 5}$ & 29.52 \\
& InceptionV2 & 68.49 & 97.77 & 29.28 \\
& EfficientNet-b0 & 66.70 & 97.27 & 30.57 \\
\hline \hline & AlexNet & 35.02 & 92.15 & 57.13 \\
& VGG16 & 72.34 & 98.50 & 26.16 \\
Stanford & ResNet18 & 63.98 & $\mathbf{9 8 . 8 1}$ & 34.83 \\
-Cars & ResNet101 & 64.66 & 97.97 & 33.31 \\
& InceptionV2 & 63.02 & 98.66 & 35.64 \\
& EfficientNet-b0 & 65.5 & 97.57 & 32.07 \\
\hline \hline & AlexNet & 46.08 & 87.99 & 41.91 \\
& VGG16 & 60.42 & 92.97 & 32.55 \\
FGVC & ResNet18 & 59.34 & $\mathbf{9 5 . 6 4}$ & 36.30 \\
-Aircraft & ResNet101 & 52.77 & 94.92 & 42.15 \\
& InceptionV2 & 57.17 & 94.80 & 37.68 \\
& EfficientNet-b0 & 51.54 & 94.89 & 43.35 \\
\hline
\end{tabular}

On CUB200-2011, the current best recognition accuracy is $90 \%$, and our proposed method based on ResNet models(ResNet18 and ResNet101) all exceed 98\%; while the lowest recognition accuracy by using LSTM-RDN (AlexNet) reaches $92.30 \%$ (AlexNet), which is 2 percentage points higher than the best method. On the Stanford Cars and FGVC-

TABLE VI

RECENT RESULTS ON CUB200-2011

\begin{tabular}{c|c|c}
\hline Model & Backbone model & Accuracy \\
\hline Our LSTM-RDN & AlexNet & 92.30 \\
Our LSTM-RDN & VGG16 & 96.27 \\
Our LSTM-RDN & ResNet18 & 98.11 \\
Our LSTM-RDN & ResNet101 & $\mathbf{9 8 . 1 5}$ \\
Our LSTM-RDN & InceptionV2 & 97.77 \\
Our LSTM-RDN & EfficientNet-b0 & 97.27 \\
\hline \hline API-Net[45] & DenseNet161 & $\mathbf{9 0 . 0 0}$ \\
MC-Loss[46] & B-CNN & 86.40 \\
DF-GMM[47] & - & 88.80 \\
PC-Softmax[43] & - & 89.73 \\
MGE-CNN[48] & Resnet-101 & 89.40 \\
DCL[49] & ResNet50 & 87.80 \\
MMAL-Net[50] & ResNet-50 & 89.60 \\
\hline
\end{tabular}

Aircraft datasets, the highest recognition accuracy accura- 
cies are 96.2\% DAT(AmoebaNet-B) and 94.70\% MMALNet(ResNet-50), respectively; while, the best recognition accuracies of LSTM-RDN (ResNet18) are $98.81 \%$ and $95.64 \%$,respectively. The specific results are shown in Table VII and Table VIII

TABLE VII

RECENT RESULTS ON STANFORD CARS

\begin{tabular}{c|c|c}
\hline Model & Backbone model & Accuracy \\
\hline Our LSTM-RDN & AlexNet & 92.15 \\
Our LSTM-RDN & VGG16 & 98.50 \\
Our LSTM-RDN & ResNet18 & $\mathbf{9 8 . 8 1}$ \\
Our LSTM-RDN & ResNet101 & 97.97 \\
Our LSTM-RDN & InceptionV2 & 98.66 \\
Our LSTM-RDN & EfficientNet-b0 & 97.57 \\
\hline \hline API-Net[45] & DenseNet161 & 95.30 \\
MC-Loss[46] & B-CNN & 94.40 \\
SpinalNet[44] & ResNet101 & 93.35 \\
DAT[38] & AmoebaNet-B & $\mathbf{9 6 . 2 0}$ \\
MGE-CNN[48] & Resnet-50 & 93.90 \\
DF-GMM[47] & - & 94.80 \\
DCL[49] & ResNet50 & 94.50 \\
MMAL-Net[50] & ResNet-50 & 95.0 \\
ResMLP-24[19] & MPL & 89.50 \\
\hline
\end{tabular}

TABLE VIII

RECENT RESULTS ON FGVC-AIRCRAFT

\begin{tabular}{c|c|c}
\hline Model & Backbone model & Accuracy \\
\hline Our LSTM-RDN & AlexNet & 87.99 \\
Our LSTM-RDN & VGG16 & 92.97 \\
Our LSTM-RDN & ResNet18 & $\mathbf{9 5 . 6 4}$ \\
Our LSTM-RDN & ResNet101 & 94.92 \\
Our LSTM-RDN & InceptionV2 & 94.80 \\
Our LSTM-RDN & EfficientNet-b0 & 94.89 \\
\hline \hline API-Net[45] & DenseNet161 & 90.30 \\
MC-Loss[46] & B-CNN & 92.90 \\
DF-GMM[47] & - & 93.80 \\
DAT[38] & InceptionV3 & 94.10 \\
DCL[49] & ResNet50 & 93.00 \\
MMAL-Net[50] & ResNet-50 & $\mathbf{9 4 . 7 0}$ \\
\hline
\end{tabular}

\section{COVID-19 prediction}

We train a binary classification model with transfer learning for predicting whether a CT image is COVID or non-COVID. The evaluation is executed on COVID_CT dataset[51] which is an internet- based dataset that contains CT images from patients. This dataset contains 349 CT images positive for COVID-19 belonging to 216 patients and 397 CT images that are negative for COVID-19. It is divided into three sets: train, validation and test sets(see Table IX). The train and validation sets are used for model learning. The test set is used to measure the recognition accuracy of the models. In order to obtain good performance, for the one-dimensional features generated by different backbone models, we use different training parameters including loss functions, learning rates and dropout probabilities to fine-tune LSTM-RDN model.

In the experiments, accuracy, precision, F1-score and recall evaluation measures were used. The calculation of these mea-
TABLE IX

DATA SPLIT IN COVID_CT DATASET 51

\begin{tabular}{c|c|c|c|c}
\hline & Totale & Train set & validation set & test set \\
\hline COVID & 349 & 191 & 60 & 98 \\
Non-COVID & 397 & 234 & 58 & 105 \\
\hline
\end{tabular}

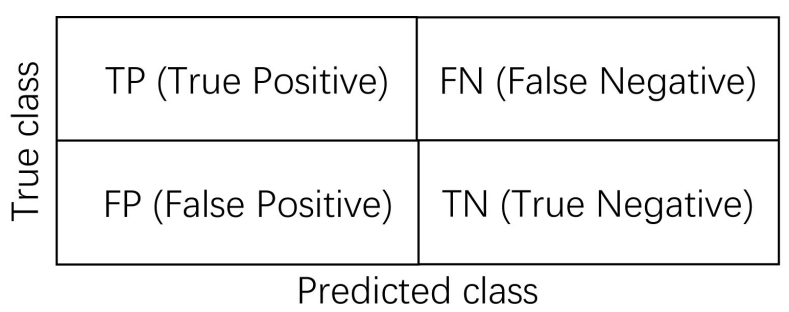

Fig. 9. The define of confusion matrix for binary classification.

sures is related to the confusion matrix. The confusion matrix for binary classification is shown in Fig 9 .

$$
\begin{aligned}
\text { Accuracy } & =\frac{T P+T N}{T P+T N+F P+F N}, \\
\text { Precision } & =\frac{T P}{T P+F P}, \\
\text { Recall } & =\frac{T P}{T P+F N}, \\
F 1-\text { score } & =2 \times \frac{\text { Precision } \times \text { Recall }}{\text { Precision }+ \text { Recall }} .
\end{aligned}
$$

TABLE $X$

THE ACCURACY ON COVID_CT(\%)

\begin{tabular}{c|c|c}
\hline Model & Backbone model & LSTM-RDN \\
\hline VGG16 & 73.39 & 95.46 \\
ResNet18 & 72.41 & 95.51 \\
ResNet101 & 75.37 & $\mathbf{9 9 . 0 1}$ \\
\hline HFSM[52] & \multicolumn{2}{|c}{93.0} \\
DenseNet-169 (mask) [51] & \multicolumn{2}{|c}{87.1} \\
TL+CSS[51] & \multicolumn{2}{|c}{86.0} \\
Multi-Task deep model[53] & \multicolumn{2}{|c}{}
\end{tabular}
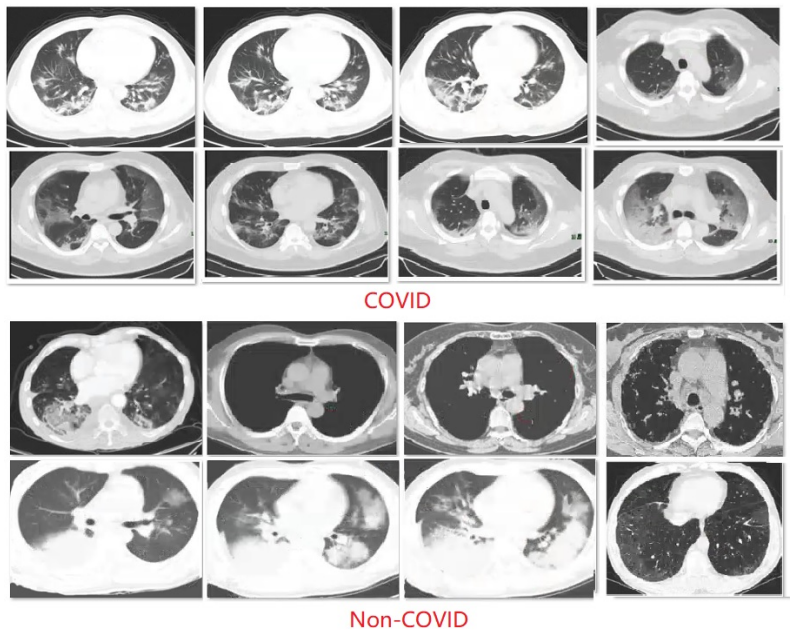

Fig. 10. Samples selected from COVID_CT dataset. The first two rows show CT images of COVID; The last two rows are CT images Non-COVID 
Table $\mathrm{X}$ shows the recognition accuracy of VGG16 is $73.39 \%$, while the performance is improved to $95.46 \%$ by feature selective transformation with LSTM-RDN. Resnet18 achieved an accuracy of $95.51 \%$, which is very close to VGG16's recognition performance. Resnet101, by contrast, performs quite well on this dataset, with a recognition rate of $99.01 \%$. While, the accuracy of DenseNet169 with mask which has more parameters and more layers is $87.1 \%$, and TL+CSS obtains $89.1 \%$ accuracy. The advanced method HFSM [52], which also uses feature selection to improve performance, achieves $93.0 \%$ accuracy. It can be seen that our method achieves the good performance on the three backbone models (VGG16,ResNet18 and ResNet101).

TABLE XI

THE OTHER EVALUATION MEASURES ON COVID_CT(\%)

\begin{tabular}{c|c|c|c}
\hline Model & Precision & Recall & F1-score \\
\hline DenseNet169(mask)[51] & - & - & 88.1 \\
TL+CSSL[51] & - & - & 89.6 \\
HFSM[52] & 72 & 71 & - \\
LSTM-RDN (VGG16) & 96.81 & 92.86 & 94.79 \\
LSTM-RDN(ResNet18) & 94.17 & $\mathbf{9 8 . 8 0}$ & 96.52 \\
LSTM-RDN(ResNet101) & $\mathbf{1 0 0}$ & 97.96 & $\mathbf{9 8 . 9 7}$ \\
\hline
\end{tabular}

In other measures, DenseNet169 (mask) and TL+CSS achieved $88.1 \%$ and $89.6 \%$ on F1-score, respectively, and our method significantly surpassed these two methods, reaching a maximum of $98.97 \%$, and increasing about 10 percent points. Although HFSM performs well in accuracy, it is relatively poor in precision and recall measures, only getting $72 \%$ and $71 \%$, respectively. LSTM-RDN(ResNet101) shows excellent performance; its precision is $100 \%$, and the recall also reaches 97.96\%. LSTM-RDN(ResNet18) and LSTM-RDN (VGG16) are also significantly better than the other three methods.

We use the confusion matrix to visually show the performance of LSTM-RDN(ResNet101), LSTM-RDN(ResNet18) and LSTM-RDN (VGG16)(see Fig.11). Only two COVID samples were misjudged as Non-COVID samples by LSTMRDN (ResNet101). LSTM-RDN (ResNet18) mistakenly judged one COVID as Non-COVID, and also mistakenly judged 6 Non-COVID samples as COVID samples. LSTMRDN (VGG16) has more errors for predicting COVID, reaching 7 samples, but it has fewer errors for predicting NonCOVID, only 2 samples.

Due to the randomness of LST-RDN, the identification results of the network of each trained model may have slight changes. We have conducted 10 times of training and testing, and the testing accuracy obtained has slight fluctuations (see Fig, 12). The fluctuation range is about $1.5 \%$. In addition to the use of Dropout, the fluctuations of recognition accuracy maybe caused by the noise in CT images and the small number of samples.

\section{DISCUSS AND ABLATION}

From Table II and Table VI, it can be seen that for ordinary object image recognition, two shallow networks AlexNet and VGG16 with LSTM-RDN have obtained better results; super-deep DCNN, such as ResNet101, InceptionV2 and EfficientNet-b0, does not have obvious advantages over shallow networks. In fine-grained image recognition, ResNets, InceptionV2 have achieved excellent results, among which the lightweight ResNet18 has outstanding performance. Therefore, we have the following conclusions:

- For coarse-grained image classification, LSTM-RDN combing with a simple or shallow network model such as AlexNet can achieve satisfactory results.

- For fine-grained image classification, LSTM-RDN combing with the ResNet models that take into account multi-scale convolution are more effective. For example, ResNet101 has best performance with more than $99 \%$ recognition accuracy for COVID-19 prediction.

- With the LSTM-RDN's feature selective transformation, the backbone network model does not need to have so many layers and branches. For example, ResNet18 with only 71 layers has equal or better performance than ResNet101 with 347 layers and EfficientNet-b0 with 290 layers, as well as multi-branch 824-layer InceptionV2 on fine-grained image classification.

- For COVID-19 prediction, the modest deep model ResNet101 with LSTM-RDN has obvious advantage than two shallow models including ResNwt18 and VGG16; Super-deep model such as InceptionV2 and EfficientNetb0 yield average performance. Lung CT image is a type of special fine-grained image which contains large amount of noise and the number of samples in CT image dataset is far more less than ordinary image datasets. This is the main cause of performance degradation of super-deep model.

LSTM-RDN contains two important parts: a LSTM layer, as well as several ReLU and Dropout layers; both parts play an important role. LSTM can increase the recognition accuracy of AlexNet/ResNet18 to more than $80 \% / 90 \%$ on the FGVCAircraft and Stanford Cars datasets, thanks to the unique memory structure of LSTM. Next, we need to know how effective the Dropout layer is in LSTM-RDN. Fig 13 and Fig 14 show the recognition performance of LSTM combined with different numbers of Dropout layers on two datasets (AlexNet and ResNet18). When the number of feature dimensions is large, more Dropout layers are needed. It also requires more training time. In the experiment, we conclude that the performance of the two Dropout layers is almost optimal based on considering the recognition accuracy and training time.

It is natural to think of using LSTM-RDN to combine the features of multiple models for further improving performance. We make two combinations including ResNet18+AlexNet and ResNet18+ResNet101 to evaluate the performance on Cifar100 (see Table XII). The experiment on Cifar100 shows that compared with a single DCNN features, the feature combination of two DCNNs only slightly improves the recognition accuracy. The advantage of combination is we can obtain more robust performance compared to single model.

\section{CONCLUSIONS}

At present, a large number of image classification models have appeared, and have achieved unprecedented performance improvements. However, they still have not broken the bottleneck of classification tasks. The parameter scale of hundreds of 

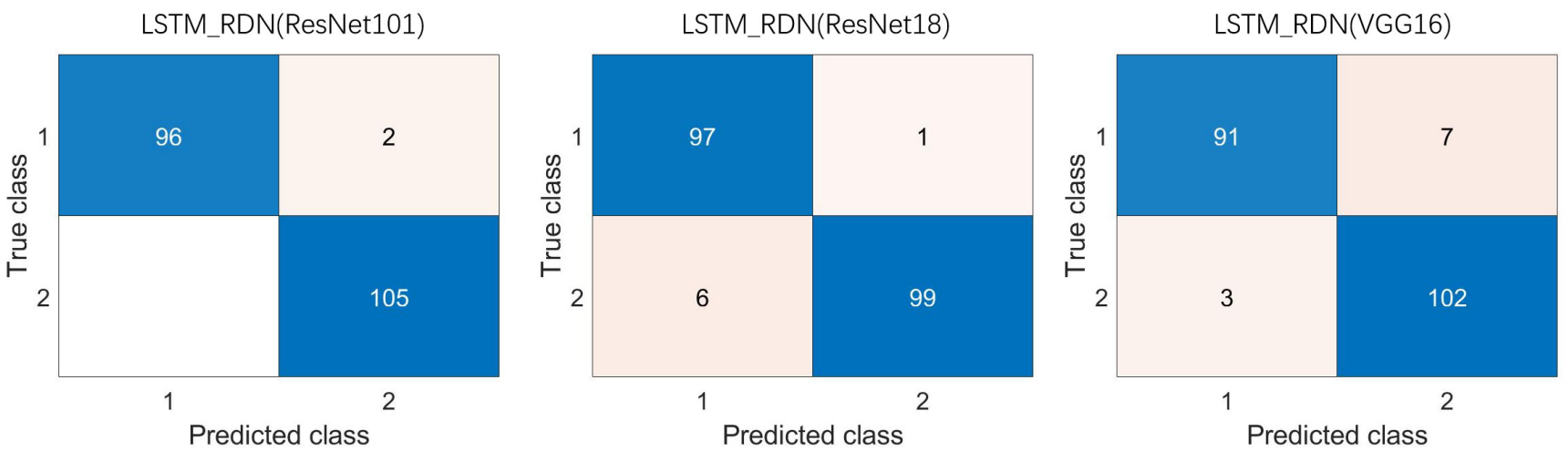

Fig. 11. Confusion matrix. Number 1 represents the COVID class, number 2 represents the Non-COVID class.

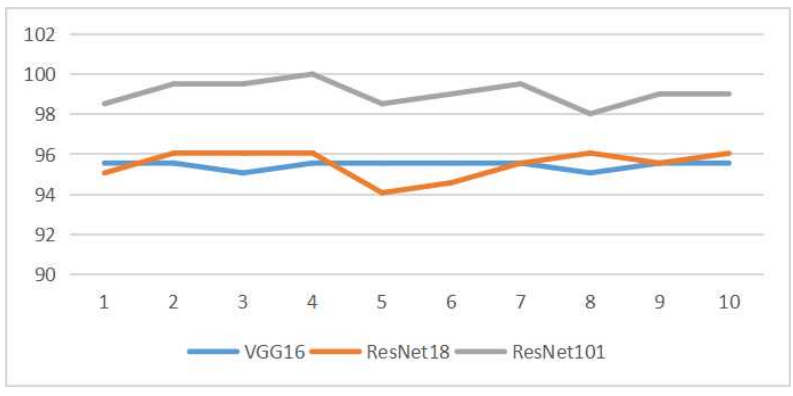

Fig. 12. The prediction accuracies of 10 times training.

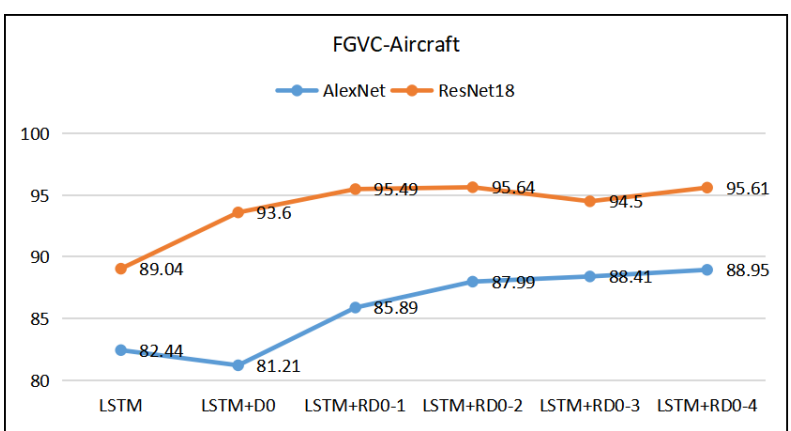

Fig. 13. The recognition performance of LSTM combining different numbers of Dropout layers on FGVC-Aircraft dataset.LSTM-D0 is LSTM combined with the first dropout, LSTM-RD0-1 denotes the LSTM layer combined with ReLU and the first Dropout layer(D0) and second Dropout layer (D1), and so on.

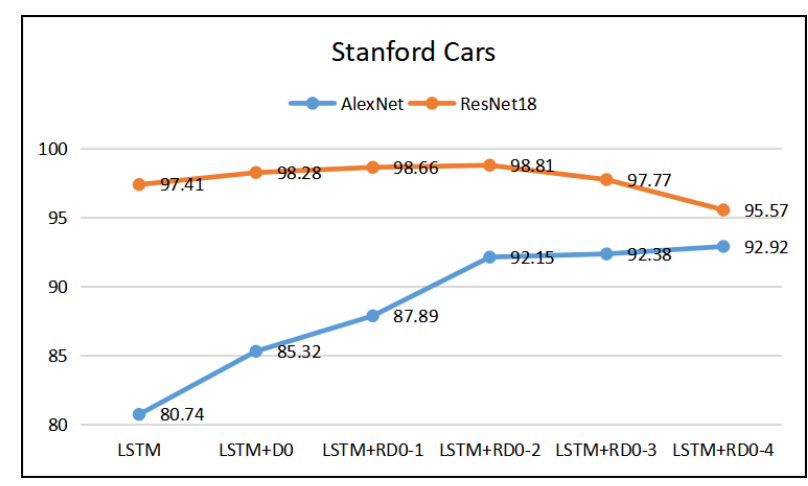

Fig. 14. The recognition performance of LSTM combining different numbers of Dropout layers on Stanford Cars dataset.
TABLE XII

PERFORMANCE COMPARISON (RECOGNITION ACCURACY) OF LSTM-RDN COMBINING MULTI-MODELS AND A SINGLE MODEL ON CIFAR 100

\begin{tabular}{c|c|c}
\hline ResNet18 & AlexNet & ResNet18+AlexNet \\
\hline 95.65 & 96.57 & 96.59 \\
\hline \hline ResNet18 & ResNet101 & ResNet18+ResNet101 \\
\hline 95.65 & 97.33 & 97.44 \\
\hline
\end{tabular}

millions is the main factor restricting performance improvement. With mainstream optimal techniques such as gradient descent algorithm, it is difficult to achieve optimal model parameters even using millions of samples and thousands of training epochs. Multi-stage processing strategy can reduce the workload of optimizing parameters exponentially, which is an effective strategy to overcome the problem of huge parameters. We have improved the classification accuracy to a new level on several image databases through a two-stage strategy (feature extraction and feature selective transformation). Experiments show that the accuracy of the backbone model network can be increased by more than $30 \%$.

Based on multi-stage strategy, we proposed LSTM-RDN, which can improve the performance of the pre-trained network models such as AlexNet and ResNet18 to a very satisfactory level. We also come to the conclusion the performance of shallow network can be even better than that of super-deep network by feature selective transformation. The LSTM-RDN's performance may have room for improvement, so, in future work, we will continue to conduct more research on the feature selective transformation, such as exploring the influence of the feature dimension, as well as the number of LSTM layers and the number of hidden units of LSTM-RDN.

\section{REFERENCES}

[1] K. He, J. Sun, and X. Tang, "Guided image filtering," IEEE Transactions on Pattern Analysis and Machine Intelligence, vol. 35, no. 6, pp. 1397 1409, 2013.

[2] J. Liu, A. Kanazawa, D. Jacobs, and P. Belhumeur, "Dog breed classification using part localization," in European Conference on Computer Vision, 2012.

[3] S. Dua, U. R. Acharya, P. Chowriappa, and S. V. Sree, "Waveletbased energy features for glaucomatous image classification," IEEE Transactions on Information Technology in Biomedicine, vol. 16, no. 1, pp. 80-87, 2011. 
[4] T. Ojala, M. Pietikainen, and T. Maenpaa, "Multiresolution gray-scale and rotation invariant texture classification with local binary patterns," IEEE Transactions on Pattern Analysis and Machine Intelligence, vol. 24, no. 7, pp. 971-987, 2002.

[5] D. G. Lowe, "Lowe, d.g.: Distinctive image features from scale-invariant key-points," International Journal of Computer Vision, vol. 60, no. 2, pp. 91-110, 2004

[6] H. Du, L. Ma, G. Li, and S. Wang, "Low-rank graph preserving discriminative dictionary learning for image recognition," Knowledge Based Systems, vol. 187, p. 104823, 2020.

[7] R. Rigamonti, A. Sironi, V. Lepetit, and P. Fua, "Learning separable filters," in 2013 IEEE Conference on Computer Vision and Pattern Recognition, 2013, pp. 2754-2761.

[8] A. Bolovinou, I. Pratikakis, and S. Perantonis, "Bag of spatio-visual words for context inference in scene classification," Pattern Recognition, vol. 46, no. 3, pp. 1039-1053, 2013.

[9] P.-S. Wang, C.-Y. Sun, Y. Liu, and X. Tong, "Adaptive o-cnn: a patch-based deep representation of $3 \mathrm{~d}$ shapes," ACM Transactions on Graphics, vol. 37, no. 6, pp. 1-11, 2019.

[10] Krizhevsky, Alex, Sutskever, Ilya, Hinton, and E. Geoffrey, "Imagenet classification with deep convolutional neural networks." Communications of the Acm, pp. 1-9, 2017.

[11] S.Karen and Z.Andrew, "Very deep convolutional networks for largescale image recognition," arXiv:1409.1556v6, 2014.

[12] B. Zoph, V. Vasudevan, J. Shlens, and Q. V. Le, "Learning transferable architectures for scalable image recognition," in CVPR, 2018, pp. 86988710.

[13] K. He, X. Zhang, S. Ren, and J. Sun, "Deep residual learning for image recognition," in CVPR, 2016, pp. 770-778.

[14] S. Zagoruyko and N. Komodakis, "Wide residual networks," 2016.

[15] S. Ioffe and C. Szegedy, "Batch normalization: Accelerating deep network training by reducing internal covariate shift," JMLR.org, 2015

[16] C. Szegedy, V. Vanhoucke, S. Ioffe, J. Shlens, and Z. Wojna, "Rethinking the inception architecture for computer vision," IEEE, pp. 2818-2826, 2016.

[17] C. Szegedy, S. Ioffe, V. Vanhoucke, and A. Alemi, "Inception-v4, inception-resnet and the impact of residual connections on learning," 2016.

[18] S. Khan, M. Naseer, M. Hayat, S. W. Zamir, and M. Shah, "Transformers in vision: A survey," arXiv:2101.01169v1.

[19] H. Touvron, P. Bojanowski, M. Caron, M. Cord, and H. Jégou, "Resmlp: Feedforward networks for image classification with data-efficient training," in CVPR, 2021.

[20] I. Tolstikhin, N. Houlsby, A. Kolesnikov, L. Beyer, X. Zhai, T. Unterthiner, J. Yung, A. Steiner, D. Keysers, J. Uszkoreit, M. Lucic, and A. Dosovitskiy, "Mlp-mixer: An all-mlp architecture for vision," arXiv:2105.01601v2.

[21] A.Dosovitskiy, L. Beyer, ander Kolesnikov, D. Weissenborn, X. Zhai, T. Unterthiner, M. Dehghani, M. Minderer, G. Heigold, S. Gelly, J. Uszkoreit, and N. Houlsby, "An image is worth $16 \times 16$ words: Transformers for image recognition at scale," arXiv:2010.11929v1, pp. $1-21,2020$

[22] C. Li, Y. Huang, and Y. Xue, "Dependence structure of gabor wavelets based on copula for face recognition," Expert Systems with Applications, vol. 137, pp. 453-470, 2019.

[23] S. Yao, S. Pan, T. Wang, C. Zheng, W. Shen, and Y. Chong, "A new pedestrian detection method based on combined hog and lss features," Neurocomputing, vol. 151, no. mar.3pt.3, pp. 1006-1014, 2015.

[24] "Multi-instance multi-label image classification: A neural approach," Neurocomputing, vol. 99, pp. 298-306, 2013.

[25] W. Jiang, Y. Yi, J. Mao, Z. Huang, and X. Wei, "Cnn-rnn: A unified framework for multi-label image classification," in 2016 IEEE Conference on Computer Vision and Pattern Recognition (CVPR), 2016.

[26] M. Yang, L. Zhang, X. Feng, and D. Zhang, "Sparse representation based fisher discrimination dictionary learning for image classification," International Journal of Computer Vision, vol. 109, no. 3, pp. 209-232, 2014.

[27] J. Ma and Y. Yuan, "Dimension reduction of image deep feature using pca," Journal of Visual Communication and Image Representation, vol. 63, p. 102578, 2019.

[28] R. Varatharajan, G. Manogaran, and M. K. Priyan, "A big data classification approach using lda with an enhanced svm method for ecg signals in cloud computing," Multimedia Tools and Applications, 2017.

[29] L. Sun, Z. Mo, F. Yan, L. Xia, F. Shan, Z. Ding, B. Song, W. Gao, W. Shao, F. Shi et al., "Adaptive feature selection guided deep forest for covid-19 classification with chest ct," IEEE Journal of Biomedical and Health Informatics, vol. 24, no. 10, pp. 2798-2805, 2020.
[30] A. K. Bhunia, A. Konwer, A. K. Bhunia, A. Bhowmick, P. P. Roy, and U. Pal, "Script identification in natural scene image and video frames using an attention based convolutional-lstm network," Pattern Recognition, vol. 85, pp. 172-184, 2019.

[31] J. Wang, L. C. Yu, K. R. Lai, and X. Zhang, "Tree-structured regional cnn-lstm model for dimensional sentiment analysis," IEEE/ACM Transactions on Audio Speech and Language Processing, vol. 28, pp. 581591,2020

[32] J. Choe, S. Lee, and H. Shim, "Attention-based dropout layer for weakly supervised single object localization and semantic segmentation," IEEE Transactions on Pattern Analysis and Machine Intelligence, vol. PP, no. 99, pp. 1-1, 2020.

[33] H. M. B. N. Pierre Foret, Ariel Kleiner, "Sharpness-aware minimization for efficiently improving generalization," arXiv:2010.01412, pp. 1-18, 2020.

[34] M. Tan and Q. V. Le, "Efficientnet: Rethinking model scaling for convolutional neural networks," arXiv:1905.11946v5, 2020.

[35] A. Kolesnikov, L. Beyer, X. Zhai, J. Puigcerver, J. Yung, S. Gelly, and N. Houlsby, "Big transfer (bit): General visual representation learning," European Conference on Computer Vision, pp. 491-507, 2019.

[36] L. Wang, S. Xie, T. Li, R. Fonseca, and Y. Tian, "Sample-efficient neural architecture search by learning action space," arXiv:1906.06832v1, 2019.

[37] Z. Lu, K. Deb, and V. N. Boddeti, "Muxconv: Information multiplexing in convolutional neural networks," in CVPR, 2020, pp. 12041-12050.

[38] V. V. S. K. Q. V. L. R. P. Jiquan Ngiam, Daiyi Peng, "Domain adaptive transfer learning with specialist models," arXiv:1811.07056v1, 2018.

[39] Y. Xiong, H. Liu, S. Gupta, B. Akin, G. Bender, P. J. Kindermans, M. Tan, V. Singh, and B. Chen, "Mobiledets: Searching for object detection architectures for mobile accelerators," arXiv, pp. 1-18, 2020.

[40] T. Ridnik, H. Lawen, A. Noy, I. Friedman, E. B. Baruch, and G. Sharir, "Tresnet: High performance gpu-dedicated architecture," arXiv:2003.13630v3, 2020.

[41] C. K. M.S.Tanveer, M. U. K.Khan, "Fine-tuning darts for image classification," arXiv:2006.09042v1, pp. 1-9, 2020.

[42] X. Sun, H. Xv, J. Dong, H. Zhou, C. Chen, and Q. Li, "Few-shot learning for domain-specific fine-grained image classification," IEEE Transactions on Industrial Electronics, vol. 68, no. 4, pp. 3588-3598, 2021.

[43] B. R. N.H.Phong, "Rethinking recurrent neural networks and other improvements for image classification," arXiv:2007.15161v1, pp. 1-18, 2020.

[44] S. J. A. K. A. A. S. N. D. S. HMD Kabir, M Abdar, "Spinalnet: Deep neural network with gradual input," arXiv:2007.03347v, pp. 1-10, 2020

[45] Y. Q. Peiqin Zhuang, Yali Wang, "Learning attentive pairwise interaction for fine-grained classification," Proceedings of the AAAI Conference on Artificial Intelligence, pp. 3130-13 137, 2020.

[46] D. Chang, Y. Ding, J. Xie, A. K. Bhunia, X. Li, Z. Ma, M. Wu, J. Guo, and Y. Z. Song, "The devil is in the channels: Mutual-channel loss for fine-grained image classification," IEEE Transactions on Image Processing, vol. 29, pp. 4683-4695, 2020.

[47] Z. Wang, S. Wang, S. Yang, H. Li, J. Li, and Z. Li, "Weakly supervised fine-grained image classification via guassian mixture model oriented discriminative learning," in CVPR, 2020, pp. 9746-9755.

[48] W. L. D. T. L Zhang, S Huang, "Learning a mixture of granularityspecific experts for fine-grained categorization," in $C V P R, 2019$, pp 8332-8340

[49] Y. Chen, Y. Bai, W. Zhang, and T. Mei, "Destruction and construction learning for fine-grained image recognition," in CVPR, 2019.

[50] G. Z. Y. L. Fan Zhang, Meng Li, "Multi-branch and multi-scale attention learning for fine-grained visual categorization," arXiv:2003.09150v3, 2020.

[51] X. Yang, X. He, J. Zhao, Y. Zhang, S. Zhang, and P. Xie, "Covid-ctdataset: A ct scan dataset about covid-19," arXiv:2003.13865, 2020.

[52] A. Wms, B. Ahr, B. Ais, and C. Ae, "A new covid-19 patients detection strategy (cpds) based on hybrid feature selection and enhanced knn classifier," Knowledge-Based Systems, vol. 205, pp. 1-18, 2020.

[53] A. Amyar, R. Modzelewski, and R. Su, "Multi-task deep learning based ct imaging analysis for covid-19: Classification and segmentation," vol. $126,2020$. 